\title{
Forced convection heat transfer inside an anisotropic porous channel with oblique principal axes: Effect of viscous dissipation
}

\author{
Moghtada Mobedi ${ }^{a}$, Ozgur Cekmer ${ }^{\mathrm{b}}$, Ioan Pop ${ }^{\mathrm{c}, *}$ \\ a Mechanical Engineering Department, Izmir Institute of Technology, Urla, 35430 Izmir, Turkey \\ ${ }^{\mathrm{b}}$ Energy Engineering Program, Izmir Institute of Technology, Urla, 35410 Izmir, Turkey

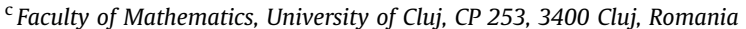

\section{A R T I C L E I N F O}

\section{Article history:}

Received 14 January 2010

Received in revised form

12 April 2010

Accepted 3 June 2010

Available online 10 July 2010

\section{Keywords:}

Anisotropic porous media

Viscous dissipation

Internal forced convection

Fully developed flow

\begin{abstract}
A B S T R A C T
An analytical study on laminar and fully developed forced convection heat transfer in a parallel-plate horizontal channel filled with an anisotropic permeability porous medium is performed. The principal axis of the anisotropic porous medium is oriented from 0 to 90 degrees. A constant heat flux is applied on the outer wall of the channel. Both clear (Newtonian) fluid and Darcy viscous dissipations are considered in the energy equation. Directional permeability ratio parameter $A^{*}$ is defined to combine both the effect of the dimensionless permeability ratio parameter $K^{*}=\left(K_{1} / K_{2}\right)$ and orientation angle $\varphi$ into one parameter. The effects of the parameter $A^{*}$, the Darcy number $\mathrm{Da}$ and the modified Brinkman number $\mathrm{Br}^{*}$ on the heat transfer and fluid flow characteristics in the channels are investigated and presented in graphs. The obtained results show that the parameters $A^{*}, D a$ and $B r^{*}$ have strong effects on the dimensionless normalized velocity and temperature profiles as well as on the Nusselt number. It is found that for a particular value of $A^{*}$, called as critical value $A_{\mathrm{cr}}^{*}$, the external heat applied to the surface of the channel is balanced by the internal heat generation due to viscous dissipation and the bulk mean temperature approaches the wall temperature. Hence, the Nusselt number approaches infinity for the critical values $A_{\mathrm{cr}}^{*}$.
\end{abstract}

(c) 2010 Elsevier Masson SAS. All rights reserved.

\section{Introduction}

Convective heat transfer in porous media is of fundamental importance to a number of technological applications, such as oil recovery, water supply management in hydrogeology, geothermal exploitation, ground heat storage, building thermal insulation, nuclear waste disposals, radioactive waste management, ground water flow modeling and is also of interest in environmental sciences and geophysics. A wide application of the porous media on many practical applications can be found in the well known books by Nield and Bejan [1], Ingham and Pop [2], Vafai [3], Pop and Ingham [4], Bejan et al. [5] and Vadasz [6], and in the recent papers by Harris et al. [7], and Kuznetsov and Nield [8]. Studies on heat transfer in porous media with forced flow in inclined and horizontal channels filled with a fluid-saturated porous medium can be found in literature. Most of the performed studies have usually been concerned with homogeneous isotropic porous structures. However, in many practical situations the porous materials are anisotropic in their mechanical and thermal properties [9]. An example of such a medium is loft insulation which usually has lower permeability

\footnotetext{
* Corresponding author. Tel.: +40 264 594315; fax: +40 264591906.

E-mail address: popm.ioan@yahoo.co.uk (I. Pop).
}

across the insulating layer than it has in the perpendicular directions. Another important example is geological systems with anisotropic sediments and rocks [10]. Within the last few years, however, the effects of non-homogeneity and anisotropy in porous media have been investigated. The inclusion of more physical realism in the matrix properties of the medium is important for the accurate modeling of the anisotropic media. Anisotropy, which is generally a consequence of a preferential orientation or asymmetric geometry of the grain or fibers, is in fact encountered in numerous systems in industry and nature. It appears that the first studies on convective flow in anisotropy porous media has been published by Castinel and Combarnous [11], who found the criterion for the onset of convection in a horizontal layer with anisotropic permeability, a problem which is the porous media analogue of the Bénard problem. They reported the experimental results which agree fairly well with their theoretical predictions. Epherre [12] extended the stability analysis by including anisotropy in the thermal diffusivity. The investigations of Castinel and Combarnous [11], and Epherre [12] were restricted to two-dimensional anisotropy, i.e. the layer was horizontally isotropic. However, a few years later Kvernvold and Tyvand [13] performed a theoretical analysis on linear and nonlinear convection in a horizontal layer where a three-dimensional anisotropy has been included. The effects of anisotropy and surface boundary conditions 


\begin{tabular}{|c|c|c|c|}
\hline \multicolumn{2}{|c|}{ Nomenclature } & $\bar{U}$ & dimensionless average velocity \\
\hline$A^{*}$ & directional normobilitu natio prometer & v & 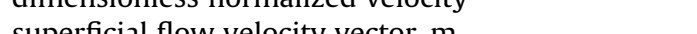 \\
\hline $\mathrm{Br}^{*}$ & modified Brinkman number & $x$ & coordinate along the axis of the channel. $m$ \\
\hline$C_{p}$ & specific heat at constant pressure, $\mathrm{J} / \mathrm{kg} \mathrm{K}$ & $y$ & coordinate normal to the surfaces of the channel, $\mathrm{m}$ \\
\hline$D a$ & Darcy number & $X, Y$ & dimensionless coordinates \\
\hline$H$ & half height of the channel, $m$ & & \\
\hline$k$ & thermal conductivity, $\mathrm{W} / \mathrm{m} \mathrm{K}$ & \multicolumn{2}{|c|}{ Greek letters } \\
\hline$K^{*}$ & dimensionless permeability ratio parameter & $\alpha$ & dimensionless anisotropic factor \\
\hline$K_{1}, K_{2}$ & peremabilities along principal axes, $\mathrm{m}^{2}$ & $\alpha_{\mathrm{m}}$ & effective thermal diffusivity, $\mathrm{m}^{2} / \mathrm{s}$ \\
\hline & second-order permeability tensor, $\mathrm{m}^{2}$ & $\phi_{\text {conv }}$ & dimensionless convective heat transport \\
\hline M & dimensionless viscosity ratio parameter & $\phi_{\text {gener }}$ & dimensionless viscous dissipation \\
\hline $\mathrm{Nu}$ & Nusselt number & $\varphi$ & orientation angle, degree \\
\hline$p$ & pressure, $\mathrm{Pa}$ & $\gamma$ & pressure gradient, Pa s/kg \\
\hline$q_{w}$ & constant wall heat flux, $\mathrm{W} / \mathrm{m}^{2}$ & $\mu_{\mathrm{f}}$ & dynamic viscosity of the fluid, $\mathrm{kg} / \mathrm{m} \mathrm{s}$ \\
\hline$T$ & temperature, $K$ & $\mu_{\text {eff }}$ & effective dynamic viscosity, kg/m s \\
\hline $\bar{T}$ & average temperature, $K$ & $\nu$ & kinematic viscosity, $\mathrm{m}^{2} / \mathrm{s}$ \\
\hline$u$ & velocity component along the $x$-direction, $\mathrm{m} / \mathrm{s}$ & $\theta$ & dimensionless temperature \\
\hline$U$ & dimensionless velocity component along $X$-direction & $\bar{\theta}_{w}$ & dimensionless average wall temperature \\
\hline $\bar{u}$ & average velocity, $\mathrm{m} / \mathrm{s}$ & $\rho$ & density, $\mathrm{kg} / \mathrm{m}^{3}$ \\
\hline
\end{tabular}

on convection in a horizontal porous layer were studied by McKibbin [14]. A very good collection of references on the topic on the effects of anisotropy on convective flow through porous media can be found in the review paper by Storesletten [9]. Further, Degan et al. [15], Zhang et al. [16], Bera et al. [17], Bera and Khalili [18], and Kumar and Bera [19] have studied the convective heat transfer in a vertical anisotropic porous layer and in a cavity filled with an anisotropic porous medium. Bera et al. [17], and Bera and Khalili [18] obtained both an analytical and numerical investigation of the double-diffusive natural convection in an anisotropic porous cavity with opposing buoyancy forces in order to understand the existence of multiple solutions and the occurrence of oscillatory convection as observed in the single component system. Degan and Vasseur [20] reported an analytical study on the aiding fully developed mixed convection in parallel-plate vertical porous channels with an anisotropic permeability whose principal axes are oriented in a direction which is oblique to the gravity vector. On the other hand, it should be also mentioned the papers by Degan et al. [21], and Vasseur and Degan [22] who have analyzed the steady natural convection over a vertical heated plate embedded in an anisotropic porous medium. Within the framework of the boundary layer approximations, similarity solutions were obtained for the case where the wall temperature varies as a power function of distance from the leading edge. Degan et al. [23] studied the transient natural convection of non-Newtonian fluids about a vertical surface embedded in an anisotropic porous medium. Finally, we mention a series of four papers by Rees and Storesletten [24], and Storesletten and Rees [25] on the effect of anisotropic permeability on free convective boundary layer flow over a vertical flat plate embedded in a porous medium. Rees and Storesletten [26] and Rees et al. [27] presented very interesting results for the free convection motions induced by point sources or horizontal line sources of heat in an anisotropic porous medium. The line source is placed at the intersection of two bounding planes.

The aim of this paper is to study the effect of viscous dissipation on the steady forced convection heat transfer inside an anisotropic porous channel with oblique principal axes using the extended Darcy-Brinkman model. In fact, the paper extends that of Hung and Tso $[28,29]$ on the temperature variations of forced convection in porous media for heating and cooling processes with internal heating effect of viscous dissipation. The analytical results were supported by numerical results to ensure of their correctness. To our best of knowledge the present problem has not been studied before, so that the results are new and original.

\section{The considered problem}

A two-dimensional parallel-plate channel filled with a porous medium saturated with an incompressible fluid that is in local thermodynamic equilibrium with the solid matrix as depicted in Fig. 1 is considered. The channel has a rectangular cross-section with height of $2 H$. It is infinitely long in the $z$-direction, $x$-being the horizontal direction of the flow and $y$-direction perpendicular to that latter. Following Degan et al. [15], we assume that the porous medium is anisotropic in flow permeability, the permeabilities along the two principal axes of the porous matrix are denoted by $K_{1}$ and $K_{2}$. The anisotropy of the porous medium is characterized by the permeability ratio $K^{*}=K_{1} / K_{2}$ and the orientation angle $\varphi$, defined as the angle between the horizontal $x$-direction and the principal axis with the permeability $K_{2}$. It is assumed that the flow in the channel is fully developed, unidirectional and steady. The effects of both clear fluid and Darcy viscous dissipations are involved in the present study. A constant heat flux $q_{w}$ is applied onto the impermeable walls of the channel.

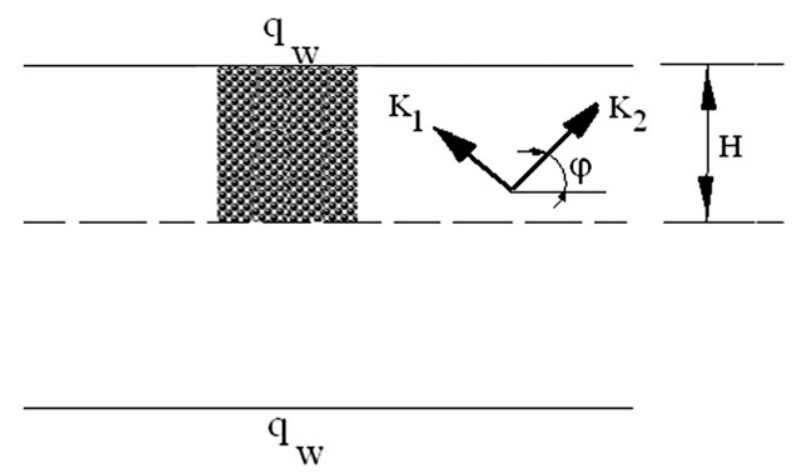

Fig. 1. Physical model of the considered channel filled with an anisotropic porous medium. 


\section{Governing equations and the boundary conditions}

The governing equations for a laminar flow in an anisotropic porous medium under assumptions explained in the preceding section are continuity, Brinkman momentum and energy, and they can be expressed as

$\nabla \cdot \mathbf{V}=0$

$\mathbf{V}=\frac{\overline{\bar{K}}}{\mu_{\mathrm{f}}}\left(-\nabla p+\mu_{\mathrm{eff}} \nabla^{2} \mathbf{V}\right)$

$\nabla \cdot(\mathbf{V} T)=\alpha_{\mathrm{m}} \nabla^{2} T+\frac{\nu}{C_{p}}\left(\frac{\partial u}{\partial y}\right)^{2}+\frac{\mu_{\text {eff }}}{\rho C_{p} K_{1}} u^{2}$

where $\mathbf{V}$ is the superficial flow velocity vector, $T$ is the fluid temperature, $p$ is the fluid pressure, $\rho$ is the fluid density, $\nu$ is the fluid kinematic viscosity, $\mu_{\mathrm{f}}$ is the fluid dynamic viscosity, $\mu_{\mathrm{eff}}$ is the effective (apparent) dynamic viscosity for Brinkman's model, $\alpha_{\mathrm{m}}$ effective thermal diffusivity of the fluid-saturated porous medium and $C_{p}$ is the specific heat at constant pressure. The first and second terms in the energy equation (Eq. (3)) show the transport of fluid in the porous medium by convection and diffusion modes, see Al-Hadhrami et al. [30]. The third term represents the effect of viscous dissipation in clear fluid flow and this expression is known for fluid flows when permeability goes to infinity and the fourth term show viscous dissipation in Darcy limit when permeability approaches to zero. The symbol $\overline{\bar{K}}$ shows a symmetrical secondorder permeability tensor which is defined as

$\overline{\bar{K}}=\left[\begin{array}{cc}K_{1} \sin ^{2} \varphi+K_{2} \cos ^{2} \varphi & \left(K_{1}-K_{2}\right) \sin \varphi \cos \varphi \\ \left(K_{2}-K_{1}\right) \sin \varphi \cos \varphi & K_{2} \sin ^{2} \varphi+K_{1} \cos ^{2} \varphi\end{array}\right]$

As it has been mentioned before, $\varphi$ is the orientation angle and the coefficients $K_{2}$ and $K_{1}$ represent permeability values along $x$ - and $y$-axes when $\varphi=0$.

\section{Solution of governing equation for the present problem}

\section{i) Solution of Brinkman momentum equation}

Since flow in the considered channel is hydrodynamically fully developed and unidirectional with $u=u(y)$, which is parallel to the $x$-axis, the following assumptions are valid;

$v=0, \quad \frac{\partial u}{\partial x}=0, \quad \frac{\partial p}{\partial y}=0$

where $v$ represents velocity in the $y$-direction which vanishes. Under the aforementioned conditions, the Brinkman moment equation (Eq. (2)) can be simplified into the following form

$\frac{\mu_{\mathrm{eff}}}{\mu_{\mathrm{f}}} \frac{\mathrm{d}^{2} u}{\mathrm{~d} y^{2}}-\frac{A^{*}}{K_{1}} u+\gamma=0$

The constant quantity $\gamma=-\left(1 / \mu_{\mathrm{f}}\right) \partial p / \partial x$ is the negative of the applied pressure gradient and the constant $A^{*}$ is the directional permeability ratio parameter, which is defined as, see Degan et al. [15],

$A^{*}=\sin ^{2} \varphi+K^{*} \cos ^{2} \varphi$

where $K^{*}=K_{1} / K_{2}$ is the permeability ratio parameter. It should be mentioned that Degan and Vasseur [20] have also considered $K_{1}$ as a reference permeability. Introducing the dimensionless variables $X, Y$ and $U$ defined as
$X=\frac{x}{H}, \quad Y=\frac{y}{H}, \quad U=\frac{u}{\gamma H^{2}} u$

the momentum Brinkman equation (Eq. (6)) can be written in dimensionless form as

$M \frac{\mathrm{d}^{2} U}{\mathrm{~d} Y^{2}}-\frac{A^{*}}{D a} U+1=0$

where $M=\mu_{\mathrm{eff}} / \mu_{\mathrm{f}}$ denotes the viscosity ratio. A non-slip wall boundary condition and symmetry condition at the centre of the channel can be employed to define boundary conditions for Eq. (9) as

$U(1)=0, \quad\left(\frac{\partial U}{\partial Y}\right)_{Y=0}=0$

The analytical solution of Eq. (9) with the boundary conditions representing by Eq. (10) can be expressed as

$U(Y)=\frac{D a}{A^{*}}\left\{\frac{1}{M}-\frac{\cosh \left[Y \sqrt{A^{*} /(M D a)}\right]}{\cosh \left[\sqrt{A^{*} /(M D a)}\right]}\right\}$

In the present study, for the sake of brevity and without loss of generality, it is assumed that $\mu_{\mathrm{eff}}=\mu_{\mathrm{f}}$ (Nield and Bejan [1]), leading to $M=1$ and Eq. (11) becomes

$U(Y)=\frac{1}{\alpha^{2}}\left[1-\frac{\cosh (\alpha Y)}{\cosh \alpha}\right]$

where $\alpha$ is the anisotropic shape factor, which is defined as

$\alpha=\sqrt{\frac{A^{*}}{D a}}$

Considering Eq. (12), the average velocities $\bar{u}$ and $\bar{U}$ can be expressed with the following expressions;

$\bar{u}=\frac{\gamma H^{2}}{\alpha^{2} \mu_{\mathrm{f}}}\left(1-\frac{\tanh \alpha}{\alpha}\right), \quad \bar{U}=\frac{1}{\alpha^{2}}\left(1-\frac{\tanh \alpha}{\alpha}\right)$

\section{ii) Solution for heat transfer equation}

For a fully developed flow in a channel with a subjected constant heat flux on its outer boundaries, the heat transfer equation can be simplified into the following form since $v=0$

$\rho C_{p} u \frac{\partial T}{\partial x}=k \frac{\partial^{2} T}{\partial y^{2}}+\mu_{\mathrm{f}}\left(\frac{\partial u}{\partial y}\right)^{2}+\frac{\mu_{\mathrm{f}}}{K_{1}} u^{2}$

where $k$ is the thermal conductivity of the porous medium. The above equation can be integrated along height of the channel as

$\rho C_{p} \frac{\partial}{\partial x} \int_{0}^{H} u T \mathrm{~d} y=\left.k \frac{\partial T}{\partial y}\right|_{0} ^{H}+\mu \int_{0}^{H}\left(\frac{\partial u}{\partial y}\right)^{2} \mathrm{~d} y+\frac{\mu}{K_{1}} \int_{0}^{H} u^{2} \mathrm{~d} y$

The constant wall heat flux boundary condition can be written as

$\left(\frac{\partial T}{\partial y}\right)_{y=H}=\frac{q_{w}}{k}$

where $q_{w}$ is positive for heating process (fluid being heated) and negative for cooling process (fluid being cooled). Another boundary condition is the symmetric condition at the centre of the channel, which is written as 
$\left(\frac{\partial T}{\partial y}\right)_{y=0}=0$

Further, we notice that for thermally fully developed flow, the temperature gradient along the axial direction $x$ can be expressed as;

$\frac{\partial T}{\partial x}=\frac{\mathrm{d} \bar{T}}{\mathrm{~d} x}$

which is independent of the transverse direction $y$. Here, $\bar{T}$ is the average temperature along height of channel. Using Eq. (12) along with Eqs. (17) and (18) yields the following equation

$\rho C_{p} \bar{u} \frac{\mathrm{d} \bar{T}}{\mathrm{~d} x}=\frac{1}{H}\left[q_{w}+(\beta+\eta) \bar{u}^{2}\right]$

where the quantities $\beta$ and $\eta$ are constants given by

$\beta=\frac{\mu_{\mathrm{f}} H}{K_{1}} \frac{\alpha\left(\alpha+2 \alpha \cosh ^{2} \alpha-3 \sinh \alpha \cosh \alpha\right)}{2(\alpha \cosh \alpha-\sinh \alpha)^{2}}$,

$\eta=\frac{\mu_{\mathrm{f}}}{H} \frac{\alpha^{3}(\sinh \alpha \cosh \alpha-\alpha)}{(\alpha \cosh \alpha-\sinh \alpha)^{2}}$

Further, we introduce the following dimensionless temperature function $\theta(Y)$ and normalized dimensionless velocity $\widehat{u}(Y)$ as

$\theta(Y)=\frac{k\left(T-T_{w}\right)}{H q_{w}}, \quad \widehat{u}(Y)=\frac{u}{\bar{u}}=\frac{U}{\bar{U}}$

Thus, by using Eqs. (20) and (22), Eq. (15) can be written as

$\frac{\mathrm{d}^{2} \theta}{\mathrm{d} Y^{2}}=\left[1+B r^{*} \frac{F(\alpha)}{D a}+B r^{*} G(\alpha)\right] \widehat{u}-B r^{*}\left[\frac{1}{D a} \widehat{u}^{2}+\left(\frac{\mathrm{d} \widehat{u}}{\mathrm{~d} Y}\right)^{2}\right]$

where $F(\alpha)$ and $G(\alpha)$ are given by

$F(\alpha)=\frac{K_{1} \beta}{\mu_{\mathrm{f}} H}=\frac{\alpha\left(\alpha+2 \alpha \cosh ^{2} \alpha-3 \sinh \alpha \cosh \alpha\right)}{2(\alpha \cosh \alpha-\sinh \alpha)^{2}}$

$G(\alpha)=\frac{H \eta}{\mu_{\mathrm{f}}}=\frac{\alpha^{3}(\sinh \alpha \cosh \alpha-\alpha)}{(\alpha \cosh \alpha-\sinh \alpha)^{2}}$

According to Kays et al. [31], the modified Brinkman number $\mathrm{Br}^{*}$ based on the constant heat flux condition can be defined as

$\mathrm{Br}^{*}=\frac{\mu_{\mathrm{f}} \bar{u}^{2}}{H q_{w}}$

The two terms of the right hand side of Eq. (23) implies the effects of the convective heat transport and internal heat generation due to viscous dissipation. In this study, these terms are shown by $\phi_{\text {conv }}(Y)$ and $\phi_{\text {gener }}(Y)$ where

$$
\begin{aligned}
& \phi_{\text {conv }}(Y)=\left[1+B r^{*} \frac{F(\alpha)}{D a}+B r^{*} G(\alpha)\right] \widehat{u} \\
& \phi_{\text {gener }}(Y)=B r^{*}\left[\frac{1}{D a} \widehat{u}^{2}+\left(\frac{\mathrm{d} \widehat{u}}{\mathrm{~d} Y}\right)^{2}\right]
\end{aligned}
$$

The dimensionless boundary conditions for Eq. (23) are

$$
\theta(1)=0, \quad\left(\frac{\partial \theta}{\partial Y}\right)_{\mathrm{Y}=0}=0
$$

The analytical solution of Eq. (23) with the boundary conditions Eq. (29) is given by

$\theta(Y)=E_{1} \cosh (2 \alpha Y)+E_{2} \cosh (\alpha Y)+E_{3} Y^{2}+E_{4}$

where $E_{1}, E_{2}, E_{3}$ and $E_{4}$ are constants given in the Appendix. In this case, the Nusselt number $N u$, based on the channel height is defined as

$N u=\frac{H q_{w}}{k\left(T_{w}-\bar{T}\right)}=-\frac{1}{\bar{\theta}_{w}}$

where $\bar{\theta}_{w}$ is the dimensionless average wall temperature. The expression for $\mathrm{Nu}$ can be found as

$$
\begin{aligned}
N u= & {[\alpha \cosh (\alpha)-\sinh (\alpha)] / } \\
& \left\{\alpha^{3}\left[P_{1} \sinh (2 \alpha)+P_{2} \sinh (3 \alpha)+P_{3} \sinh (\alpha)+P_{4}\right]\right\}
\end{aligned}
$$

where the expressions for the coefficients $P_{1}, P_{2}, P_{3}$ and $P_{4}$ are given in the Appendix and $\mathrm{Nu}$ is a function of $\mathrm{Da}, \mathrm{Br}^{*}$ and $A^{*}$.

\section{Results and discussion}

The solution of differential equation depends on the directional anisotropy parameter $A^{*}$, Darcy number $D a$ and the modified Brinkman number $\mathrm{Br}^{*}$. There is no need to discuss the effects of permeability ratio parameter, $K^{*}$, and orientation angle parameter, $\varphi$, separately since they are represented by directional permeability ratio $A^{*}$. In this section, the effects of $A^{*}, D a$ and $B r^{*}$ parameters on the velocity and temperature profiles as well as the Nusselt number are explained. As it was mentioned before, the solution of Eq. (23) under the boundary conditions Eq. (29) were both analytically and numerically found in very good agreement with each others for the all results presented in this study.

Although, the considered problem is about the analyzing of heat and fluid flow in a channel filled with an anisotropic porous material when a constant heat flux is applied on its wall, but the constant heat flux boundary condition is converted to the isothermal boundary conditions given by Eq. (29) and by using dimensionless variables and transforming of the energy equation (15) into a dimensionless ordinary differential equation (23). There are two terms on the right side of Eq. (23), which show the effects of local convective heat transfer in the flow direction (i.e. $\left.\phi_{\text {conv }}(Y)\right)$ and local viscous dissipation $\left(\phi_{\text {gener }}(Y)\right)$, respectively. The dimensionless temperature inside the channel $\theta(Y)$ depends on the difference of $\phi_{\text {conv }}(Y)-$ $\phi_{\text {gener }}(Y)$. If the value of $\phi_{\text {conv }}(Y)-\phi_{\text {gener }}(Y)$ is positive for a channel, then $\theta(Y)$ will take negative values. However; the values of $\theta(Y)$ will be positive if the values of $\phi_{\text {conv }}(Y)-\phi_{\text {gener }}(Y)$ for a channel are negative. For partially positive and negative values of $\phi_{\text {conv }}(Y)-$ $\phi_{\text {gener }}(Y)$, positive and/or negative values of $\theta(Y)$ in the channel is expected. For positive values of modified Brinkman number (i.e. $\mathrm{Br}^{*}>0$ ), negative values of dimensionless temperature indicates that the temperature of fluid flowing through the channel is smaller than the wall temperature and vice versa is valid. For $B r^{*}<0$, the negative values of $\theta(Y)$ show that wall temperature is less than the temperature of fluid inside the channel and again vice versa is valid.

Variations of the directional permeability ratio $A^{*}$ with the orientation angle $\varphi$ from 0 to 90 degrees for different values of the permeability ratio $K^{*}$ from 0.1 to 10 are shown in Fig. 2. Based on Eq. (7), the value of $A^{*}$ becomes equal to $K^{*}$ for zero orientation angle $\varphi=0$. When $\varphi=0$, the small values of $A^{*}$ (i.e. $A^{*}<1$ ) signifies relatively high permeability in the flow direction since $K_{2}>K_{1}$, however high values of $A^{*}$ (i.e. $A^{*}>1$ ) implies relatively lower permeability in the $X$-direction since $K_{2}<K_{1}$. The directional permeability ratio takes value of 1 (i.e. $A^{*}=1$ ) for identical values of $K_{2}$ and $K_{1}$. By increasing 


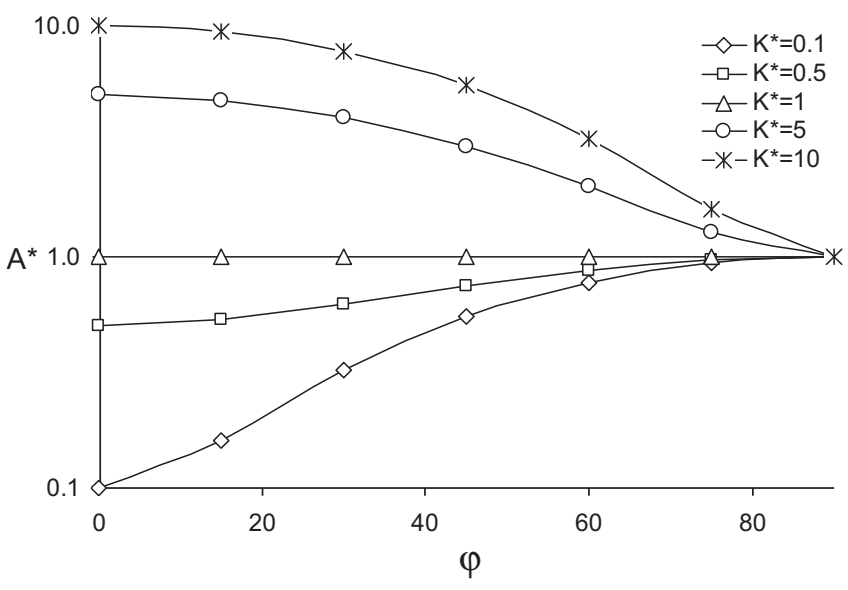

Fig. 2. Variations of the directional permeability ratio $A^{*}$ with the orientation angle $\varphi$ for different values of $K^{*}$ parameter.

the orientation angle $\varphi$ from 0 to 90 degree, three different trends for variation of $A^{*}$ with $\varphi$ are observed in Fig. 2. For $A^{*}<1$, the increase of orientation angle increases the value of directional permeability ratio and it attains to $A^{*}=1$ for $\varphi=90$ degree. Therefore, the increase of orientation angle reduces permeability in flow direction for $A^{*}<1$. For the cases with $A^{*}>1$, the increase of orientation angle reduces the value of $A^{*}$ and it falls to $A^{*}=1$ for $\varphi=90$ degree. For these cases, the increase of orientation angle increases permeability in the $X$-direction. For the cases with $A^{*}=1$, the value of the directional permeability ratio does not depend on orientation angle and heat transport in flow direction is not changed with $\varphi$.

Fig. 3 shows the variation of the dimensionless normalized velocity profiles $\widehat{u}(Y)$ for three different values of directional permeability parameter as $A^{*}=0.1,1$ and 10 and for different values of the Darcy number $D a$ from $D a=0.01$ to 10 when $B r^{*}=1$. It is seen that for $A^{*}=0.1$, the value of $\widehat{u}(Y)$ in the center of the channel increases with $D a$ since a flow in a porous medium with a high values of $D a$ approaches to clear fluid flow (Newtonian flow). That is why an obvious difference between the profiles of $\widehat{u}(Y)$ of $D a=10$ and $D a=0.01$ can be seen from Fig. 3(a). Further, as can be seen from Fig. 3(b) and (c), the profile of $\widehat{u}(Y)$ is not changed for $D a=10$ if the value of $A^{*}$ is increased from 0.1 to 1 or 10 . However, the shape of $\widehat{u}(Y)$ profiles for the low values of $D a($ e.g. $D a=0.01)$ is changed noticeably and the velocity profiles become flatten in the most part of cross-section of channel. This is an expected variation for $\widehat{u}(Y)$ profiles since when $A^{*}<1$ and it increases from 0.1 to 1 , the permeability $K_{2}$ of the porous medium in the horizontal direction of the channel decreases. We notice a steep gradient of $\widehat{u}(Y)$ at the region close to the channel walls for higher values of $A^{*}=10$ when $D a$ is low (e.g. $D a=0.01$ ).

The dimensionless convective heat transfer (i.e., $\left.\phi_{\text {conv }}(Y)\right)$ and dimensionless viscous dissipation (i.e., $\left.\phi_{\text {gener }}(Y)\right)$ profiles for three different values of $A^{*}$ when $B r^{*}=1$ are shown in Fig. 4 . As it can be seen from Fig. 4(a), the convective transport of heat at the center region of the channel with the Darcy number as $D a=0.01$ is considerably higher than that with larger Darcy number (e.g. $D a=10)$ when $B r^{*}=1$ and $A^{*}=0.1$. Fig. 4(b) shows that by increasing the value of the parameter $A^{*}$ from 0.1 to $A^{*}=1$, the convective heat transport $\phi_{\text {conv }}(Y)$ for $D a=10$ is not highly changed. However a considerable change in profile of $\phi_{\text {conv }}(Y)$ for $D a=0.01$ can be observed. The value of $\phi_{\text {conv }}(Y)$ at the center region of channel is lowered and the profiles $\phi_{\text {conv }}(Y)$ become flatten due to increase of permeability in the traverse direction. Further increase of $A^{*}$ from 1 to 10 increases permeability $K_{1}$, and the profiles of $\phi_{\text {conv }}(Y)$ take uniform shapes in the most region of the channel (Fig. 4(c)). Fig. 4(d)

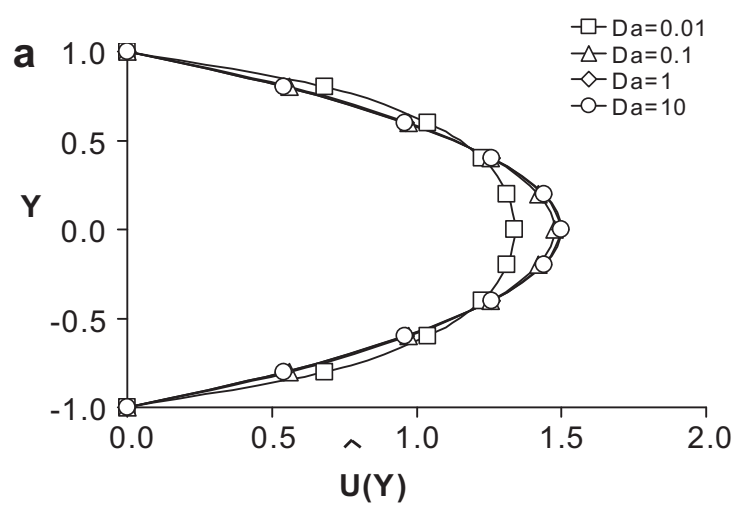

b $10 \square-\mathrm{Da}=0.01$
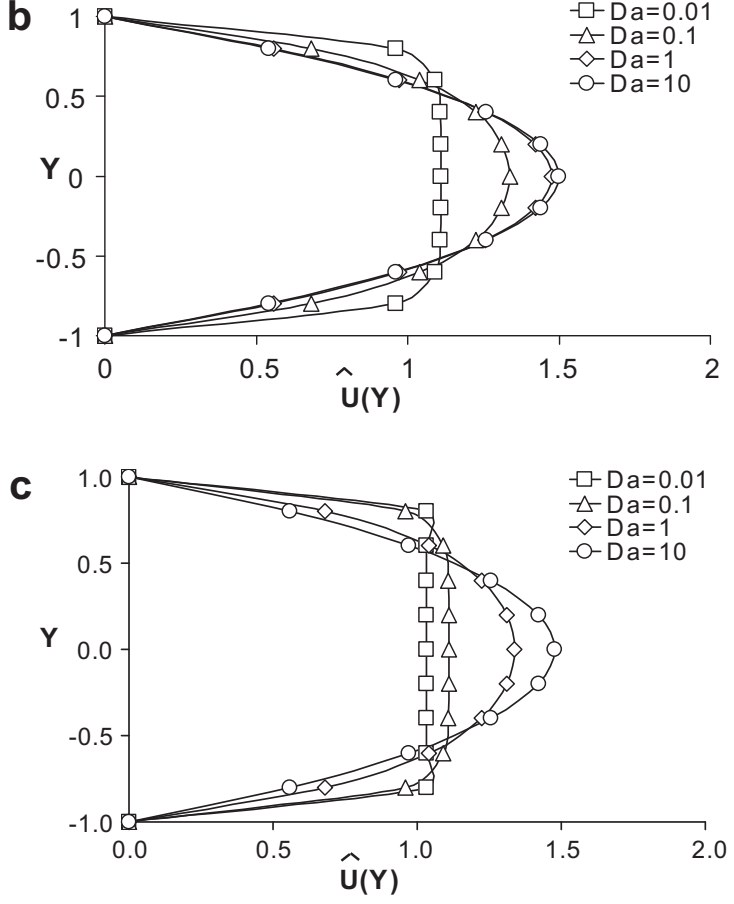

Fig. 3. Normalized velocity profiles $\widehat{u}(Y)$ in the channel for different values of $D a$ when $B r^{*}=1$ : a) $A^{*}=0.1$; b) $A^{*}=1$; c) $A^{*}=10$.

also shows that the local viscous dissipation profiles $\phi_{\text {gener }}(Y)$ in flows with high values of Darcy number (e.g. $D a=10$ ) is not large when it is compared to those with low values of $D a$ such as $D a=0.01$. The decrease of $D a$ considerably enhances viscous dissipation, particularly at the center of the channel for the flow with $\mathrm{Br}^{*}=1$ and $A^{*}=0.1$. Therefore a significant difference between profiles of $\phi_{\text {gener }}(Y)$ for $D a=10$ and 0.01 can be observed from Fig. 4(d). By increasing of directional permeability parameter $A^{*}$ from 0.1 to 1 , the viscous dissipation for channel with $D a=10$ is not highly changed. However a significant change on viscous dissipation in the channel with $D a=0.01$ occurs (Fig. 4(e)). The local viscous dissipation profile $\phi_{\text {gener }}(Y)$ near the wall is highly increased due to steep gradient of velocity $\widehat{u}(Y)$ in that region. The value of $\phi_{\text {gener }}(Y)$ is almost uniform at the center region of channel since a uniform velocity distribution $\widehat{u}(Y)$ exists in this region for $D a=0.01$. The distribution of viscous dissipation of $A^{*}=1$ (Fig. 4(e)) shows a transition behavior between the cases of $A^{*}=0.1$ and $A^{*}=10$. The velocity gradient at the wall surface is high due to increase of $A^{*}$ value and the magnitude of velocity in the center of channel is also considerable. That is why the viscous dissipation in the wall and center regions of the channel is high. This causes a double hump in distribution of viscous dissipation in the channel for low values of Darcy (Fig. 4(e)). Again, the 

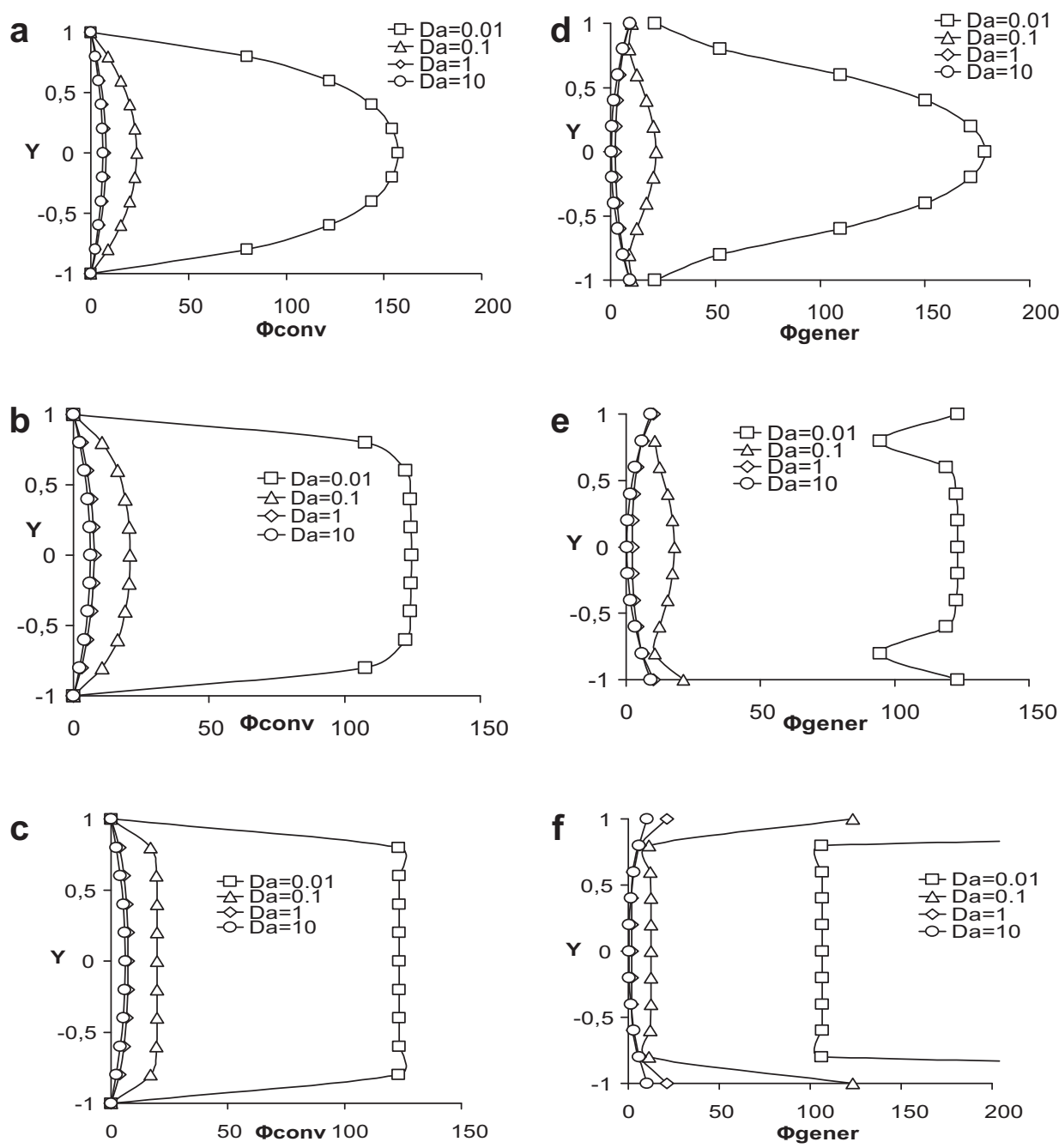

Fig. 4. Dimensionless convective transport $\phi_{\text {conv }}(Y)$ and viscous dissipation $\phi_{\text {gener }}(Y)$ profiles for different values of $D a$ when $B r^{*}=1:($ a,d $) A^{*}=0.1 ;(b, e) A^{*}=1$; $($ c,f $) A^{*}=10$.

distribution of $\phi_{\text {gener }}(Y)$ for $D a=10$ is not changed by increasing the value of $A^{*}$ from 1 to 10 as seen from Fig. 4(f). For $D a=0.01$, a considerable enhancement of $\phi_{\text {gener }}(Y)$ is observed at the region close to channel wall. The value of $\phi_{\text {gener }}(Y)$ for $D a=0.01$ at the wall surface is 1066, which is very large such that it could not be shown in Fig. $4(\mathrm{f})$. For $A^{*}=10$, the value of $\Phi_{\text {gener }}$ at the center of the channel becomes smaller compared to the value of $\Phi_{\text {gener }}$ for $A^{*}=0.1$ due to the increase of permeability ratio in the traverse direction of the channel.

Fig. 5 shows the dimensionless temperature profiles $\theta(Y)$ for values of the Darcy number $D a$ from 0.01 to 10 and for the parameter $A^{*}$ as $A^{*}=0.1,1$ and 10 when $B r^{*}=1$. The positive values of $B r^{*}$ refers to the heating of channel and therefore it is expected that the sign of $\theta$ $(Y)$ be negative since the wall temperature (i.e. $T_{w}$ ) is greater than the temperature of the fluid. For $B r^{*}=1$ and $A^{*}=0.1$ (Fig. 5(a)), when the channel is heated, the temperature difference between the fluid and the impermeable wall is negative for $D a=0.1,1$ and 10 . The absolute value of the dimensionless temperature (i.e. $|\theta(Y)|)$ for $D a=10$ is higher than $|\theta(Y)|$ in the channels with $D a=1$ and 0.1 due to higher values of $\phi_{\text {conv }}(Y)$ and low values of $\Phi_{\text {gener }}$. As it was mentioned before, heat generation due to viscous dissipation $\Phi_{\text {gener }}$ increases with decrease of $D a$ number. The increase of internal heat generation for flow $\Phi_{\text {gener }}$ with $D a=0.01$ is such that the temperature of the fluid in channel exceeds the wall temperature when $A^{*}=0.1$ and $B r^{*}=1$ as it can be observed from Fig. 5(a). That is why, the dimensionless temperature $\theta(Y)$ for $D a=0.01$ takes positive values when $A^{*}=0.1$. Fig. 4(b) shows the temperature profiles $\theta(Y)$ in the channel for $B r^{*}=1$ when $A^{*}=1$. The increase of directional permeability ratio $A^{*}$ causes the decreases of viscous dissipation in the most region of the channel and consequently the sign of the dimensionless temperature which is positive at the center of the channel for $A^{*}=0.1$ and $D a=0.01$ changes its sign and becomes negative for $D a=0.01$ referring to heating of fluid from walls. Further increase of $A^{*}$ from 1 to 10 changes the form of the temperature profiles $\theta(Y)$ for low values of $D a$ but not their signs. The largest temperature difference between fluid and wall is seen for $D a=0.01$ since the viscous dissipation (i.e. heat generation) in the wall regions considerably increases when $A^{*}$ rises from 1 to 10 .

Before explanation of the variation of the dimensionless temperature profiles $\theta(Y)$ for negative values of the modified Brinkman number $B r^{*}$, Fig. 6 is presented to understand the variations of $\phi_{\text {conv }}(Y)$ and $\phi_{\text {gener }}(Y)$ when $B r^{*}<0$. The negative values of $B r^{*}$ refer to the cooling of the channel from the outer surface and that is why both the values of $\phi_{\operatorname{conv}}(Y)$ and $\phi_{\text {gener }}(Y)$ should be negative. Fig. 6 showing the distribution of $\phi_{\text {conv }}(Y)$ and $\phi_{\text {gener }}(Y)$ for from $D a=0.01$ and 10 , and for three different values of $A^{*}$ when $B r=-1$. For $B r^{*}=-1, A^{*}=0.1$ and $D a=10$, the viscous dissipation at the walls is relatively higher compared to values of $\phi_{\text {gener }}(Y)$ at the center of the channel, as can seen from Fig. 6(a). For the flow with $D a=10$, high heat generation at the wall may balance heat extraction from 
a

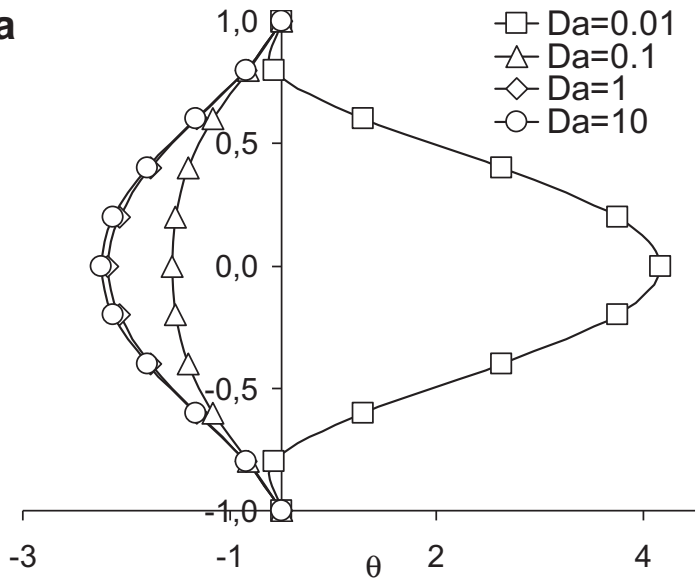

b
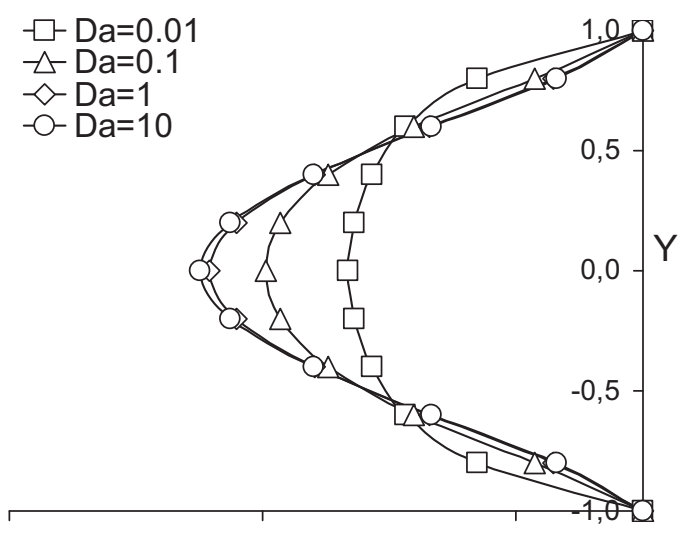

$-3$

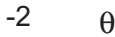

C

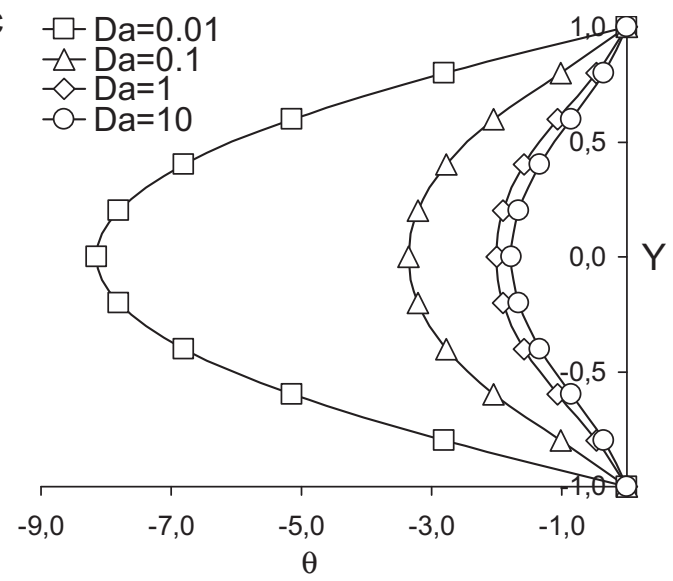

Fig. 5. Dimensionless temperature profiles $\theta(Y)$ for different values of $D a$ when $B r^{*}=1$ : (a) $A^{*}=0.1$; (b) $A^{*}=1$; (c) $A^{*}=10$.

walls and temperature at the center of wall becomes smaller than wall temperature. Fig. 6(a) also shows that the viscous dissipation at the center of the channel is higher than $\phi_{\mathrm{conv}}(Y)$ at the same region for $D a=0.01$. By increasing the value of $A^{*}$ to 1 , the distribution of $\phi_{\text {conv }}(Y)$ and $\phi_{\text {gener }}(Y)$ are not highly changed for $D a=10$, while viscous dissipation in the center of channel becomes smaller when $D a=0.01$ (Fig. 6(b)). Further increase of $A^{*}$ form 1 to 10 causes the viscous dissipation becomes smaller than $\phi_{\text {conv }}(Y)$ at the most region of the channel except at the wall region in where a considerably steep gradient of velocity exists (Fig. 6(c)). a
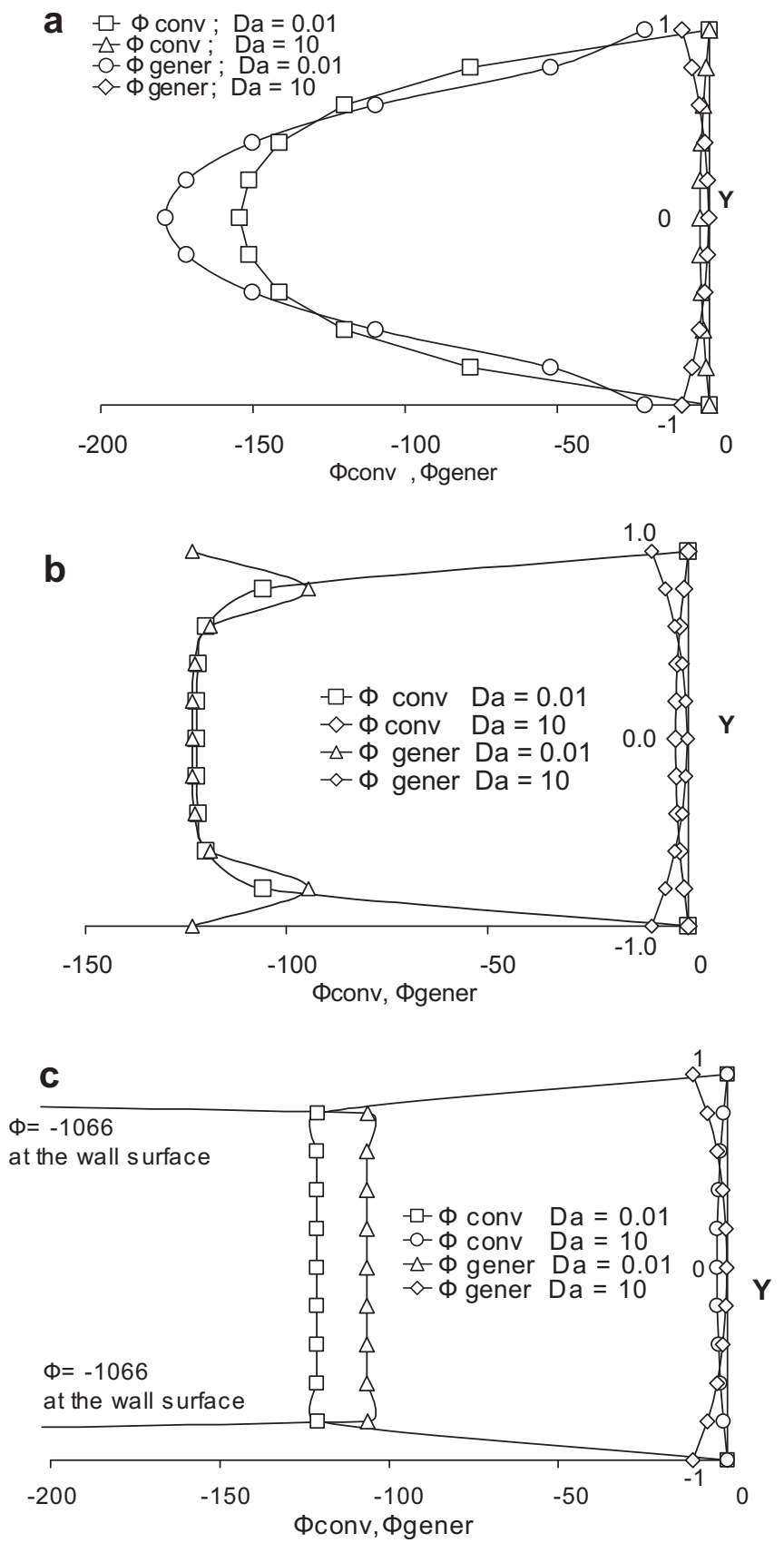

Fig. 6. Dimensionless convective transport $\phi_{\text {conv }}(Y)$ and viscous dissipation $\phi_{\text {gener }}(Y)$ profiles for two values of $D a$ when $B r^{*}=-1$ : (a) $A^{*}=0.1$; (b) $A^{*}=1$; (c) $A^{*}=10$.

The change of dimensionless temperature profile for $B r^{*}=-1$ and for the three different values of directional permeability ratios $A^{*}$ are shown in Fig. 7. The temperature in the channel depends on the difference between two negative values of $\phi_{\text {conv }}(Y)$ and $\phi_{\text {gen- }}$ er $(Y)$. For negative values of $\mathrm{Br}^{*}$, the value of the dimensionless temperature $\theta(Y)$ is expected to be negative since $q_{w}<0$ and $T_{w}<T$. However, for $B r^{*}=-1$ and $A^{*}=0.1$, and for high values of Darcy number (e.g. $D a=10$ ), the value of $\theta(Y)$ is positive at the center of the channel due to high viscous dissipation at the region close to the wall. This causes the temperature at the wall region becomes higher than the temperature of the fluid at the center region and dimensionless temperature takes positive values. For $D a=0.01$, a larger viscous dissipation at the center of channel causes a heat transfer from the center towards the walls and $\theta(Y)$ takes negative 
a

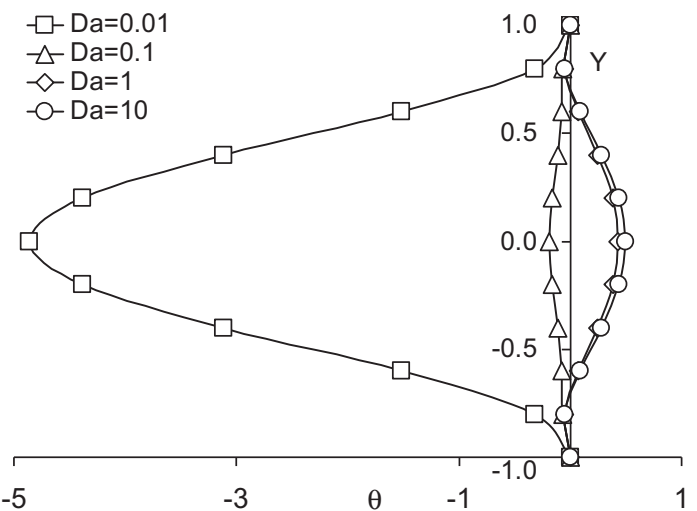

b
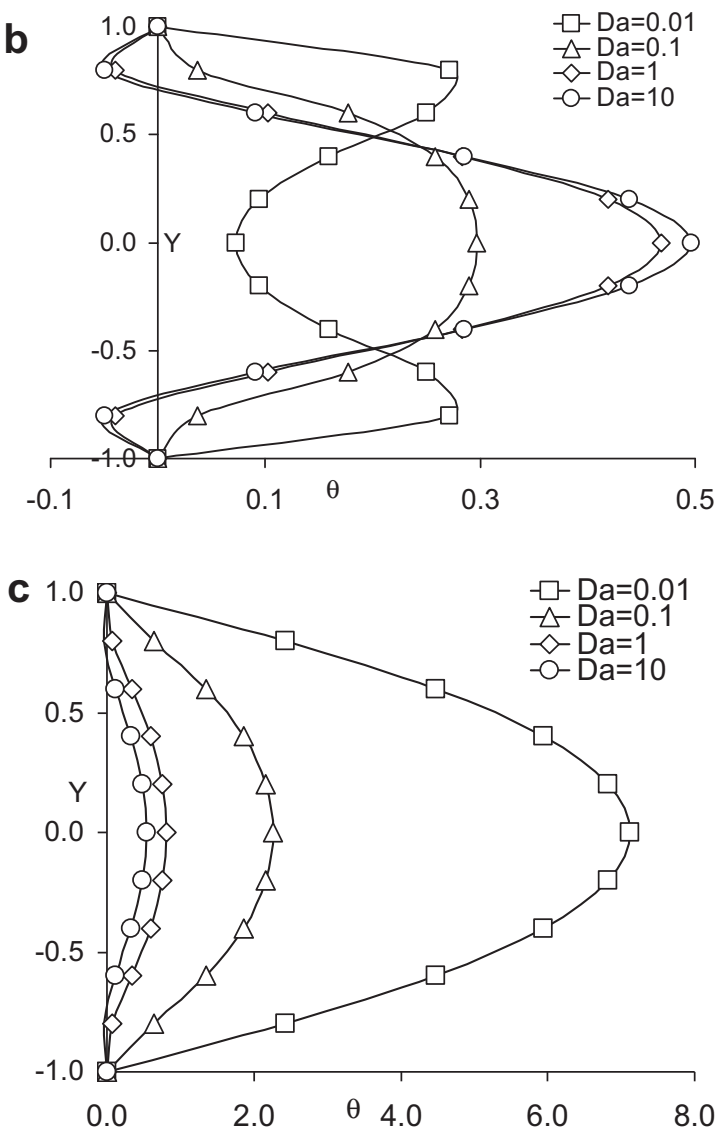

Fig. 7. Dimensionless temperature profiles $\theta(Y)$ for different values of $D a$ when $B r^{*}=-1$ : (a) $A^{*}=0.1$; (b) $A^{*}=1$; (c) $A^{*}=10$.

values. By increasing the value of $A^{*}$ from 0.1 to 1 , the shape of $\theta(Y)$ for $D a=10$ is not changed while $\theta(Y)$ for $D a=0.01$ takes positive values due to decrease of the viscous dissipation at the center and increase of heat generation at the wall region. The interesting point of Fig. 6(b) is the slope of the dimensionless temperature $\theta(Y)$ at the wall is changed and become positive for $D a=0.01$ signifying the change of direction of heat transfer at the wall. Further increases of $A^{*}$ to 10 causes the viscous dissipation is considerably increases at the wall regions while the value of $\Phi_{\text {gener }}$ is reduced at the center of channel when $D a=0.01$. That is why the temperature difference between wall and fluid is positive for $D a=0.01$ and it is higher than the value of $\theta(Y)$ for $D a=10$. As a result, for the cases with $B r^{*}<0$ and low value of $D a$ number, large heat generation particularly at the surface of the channel (Fig. 6(b) and (c)) increases the temperature of wall such that the wall temperature becomes greater than fluid flows at the center region of the channel. Thus, the direction of heat transfer is changed and heat is transferred from the wall surface to the fluid flows inside the channel. That is why the sign of slope of temperature profile at the wall is changed.

Fig. 8 shows the variation of the Nusselt number $N u$ with $A^{*}$ for $B r^{*}=1$ and -1 when $D a$ is changed from 0.01 to 10 . As it is seen from Fig. 8(a), for $D a=0.01$ and $B r^{*}=1$, a singularity is seen at $A^{*}=A_{\mathrm{cr}}^{*}=0.517$ and the sign of $N u$ is changed at this value. The value of $A^{*}$ at which the sign of $N u$ is changed can be called as critical directional permeability ratio (i.e. $A_{\mathrm{cr}}^{*}$ ). The value of $A_{\mathrm{cr}}^{*}$ depends on $\mathrm{Da}$ and $\mathrm{Br}^{*}$. The physical significance of $A_{\mathrm{Cr}}^{*}$ is that the direction of heat transfer is changed. For $D a=0.01$ and $B r=1$, the slope of the dimensionless temperature gradient in the traversal direction of the channel is changed from negative to positive values at $A_{\mathrm{cr}}^{*}=0.517$. For $A^{*}<0.517$, the value of $N u$ is negative signifying that the fluid temperature is higher than the wall temperature due to high viscous dissipation. By increasing of $A^{*}$, the sign of $N u$ is changed implying that the fluid temperature becomes smaller than the wall temperature. By increasing $D a$ from 0.01 to 0.1 , the singularity disappears and $N u$ decreases with increase of $A^{*}$. For higher values of $D a$ as $D a=1$ and 10 , the value of $N u$ is not highly changed with $A^{*}$. A similar behavior is seen for $B r^{*}=-1$, but the values of $A^{*}$ at which singularity for $N u$ exists are changed. For $B r^{*}=-1$, a singularity is seen at $A_{\mathrm{cr}}^{*}=0.895$ for $D a=0.01$. The another singularity in $\mathrm{Nu}$ for $D a=0.1$ and $B r^{*}=-1$ is observed at $A_{\mathrm{cr}}^{*}=0.41$. Further increase in $\mathrm{Da}$ causes elimination of singularity and $\mathrm{Nu}$ does not highly change with $A^{*}$ for $D a=10$ when $B r^{*}=-1$.

The variation of $A_{\mathrm{Cr}}^{*}$ with $D a$ illustrated in Fig. 9 for some values of $\mathrm{Br}^{*}$. For the cases with $B r^{*}>0$, the range of $D a$ in which the singularities occur is enlarged by increasing the values of $\mathrm{Br}^{*}$. As it is well known, the internal heat generation in the channel enhances by increasing the values of $\mathrm{Br}^{*}$ and this is why the possibility of compensation of heat flux applied to the channel by internal heat
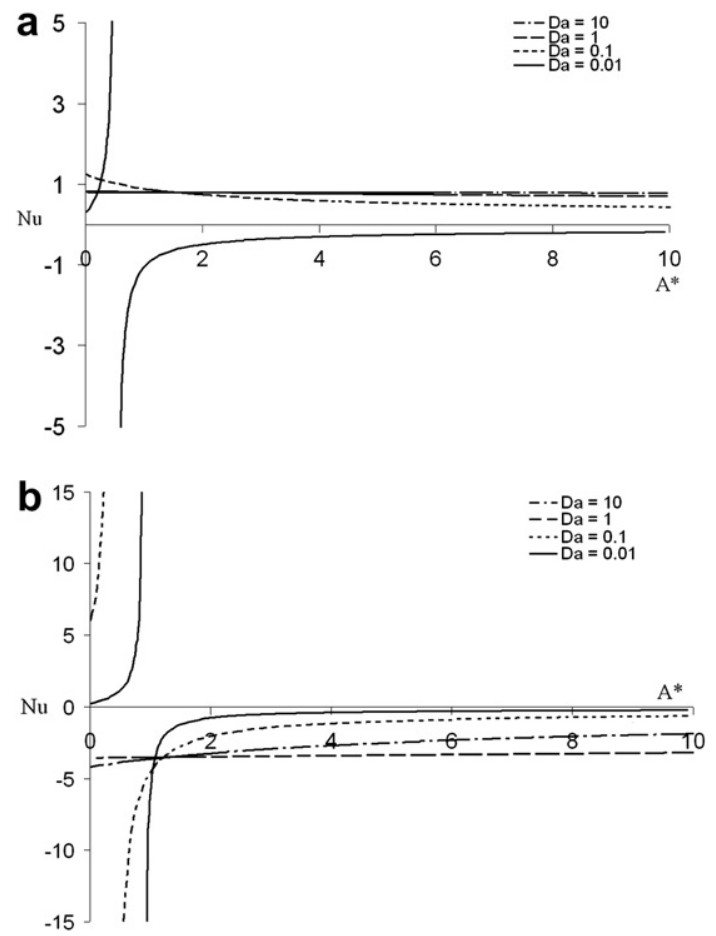

Fig. 8. Variations of the Nusselt number $N u$ with $A^{*}$ for different values of $D a$ : a) $B r^{*}=1$; b) $B r^{*}=-1$. 


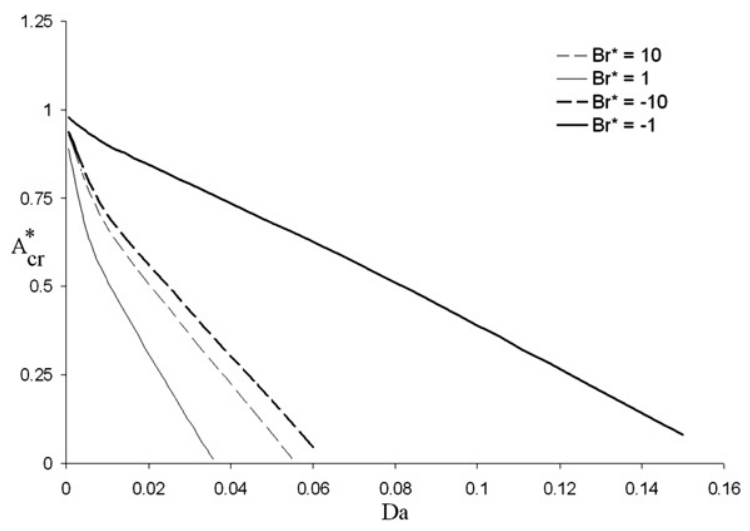

Fig. 9. Variation of $A^{*}$ with $D a$ for different values of $B r^{*}$.

generation rises. For $\mathrm{Br}^{*}<0$, implying cooling of the channel, the change of the sign of $\mathrm{Nu}$ occurs at wider range of $\mathrm{Da}$. By decreasing the values of $\mathrm{Br}^{*}$, the range of $\mathrm{Da}$ at which singularities in $\mathrm{Nu}$ occur is enlarged. The chance of balancing the heat flux in the channel with internal heat generation for the case when $\mathrm{Br}^{*}<0$ is greater compared to the cases when $\mathrm{Br}^{*}>0$, particularly for high negative modified Brinkman number.

It should be mentioned that velocity distribution in a channel filled with anisotropic flow is very different than the distribution of velocity in a channel with isotropic porous media. For the flow in an anisotropic channel when directional permeability ratio (i.e. $A^{*}$ ) is high, a uniform velocity distribution at the most region of the channel except wall region occurs while a steep velocity gradient forms at the wall region. The large velocity gradient at the wall surface generates high rate of viscous dissipation (or great generation). However, a small rate of heat generation exists in the center region of channel, as seen from Fig. 6(c). For $\mathrm{Br}^{*}<0$, not only the cooling heat flux at the surface of the wall is balanced with rate of generated heat at the wall region but also the wall temperature may exceed the temperature of fluid flows in the channel. Hence, the main reason of change of heat transfer direction is anisotropy state of porous media which causes very large velocity gradient at the wall of the channel when value of $A^{*}$ is high.

\section{Conclusion}

A study is performed to investigate the mechanism of heat and fluid flow in a channel with internal heat generation due to viscous dissipation for constant heat flux boundary condition. Heat and fluid flow are assumed fully developed in the channel. Directional permeability parameter $A^{*}$ is defined to combine the effects of permeability ratio parameter $K^{*}$ and orientation angle $\varphi$ and contribute their effect into one parameter. The parameter $A^{*}$ has a very important effect on the mechanism of heat and fluid flow in the channel. The increase of the value of $A^{*}$ enhances permeability $K_{1}$ in the traverse direction of the channel and causes the velocity profiles become flatten in the $Y$-direction. By increase of the value of $A^{*}$, high velocity gradient appear at the region close to the channel wall and this is why high internal heat generation is observed at regions close to the walls. The internal heat generation at the walls for high values of $A^{*}$ (e.g. $A^{*}=10$ ) is such that the sign of $N u$ is changed when $B r^{*}<0$. The variation of $N u$ with $A^{*}$ for different values of $D a$ shows that there are singularities in $N u$ at which the sign of heat transfer is changed. Singularities in $\mathrm{Nu}$ depends on modified Brinkman and Darcy numbers as well as on the directional permeability parameter. For $\mathrm{Br}^{*}<0$, signifying cooling of the channel, singularities are observed in $\mathrm{Nu}$ for a wide range of values of $\mathrm{Da}$.

\section{Acknowledgement}

The authors wish to express their very sincere thanks to the reviewers for their valuable comments and suggestions, which led to the substantial improvement of the paper.

\section{Appendix}

The dimensionless temperature distribution $\theta(Y)$ is found to be given by

$\theta(Y)=E_{1} \cosh (2 \alpha Y)+E_{2} \cosh (\alpha Y)+E_{3} Y^{2}+E_{4}$

where the expressions for $E_{1}, E_{2}, E_{3}, E_{4}$ and $E_{5}$ are

$$
\begin{aligned}
E_{1}= & -\frac{B r^{*}}{8 D a} \frac{\alpha^{2} D a+1}{(\alpha \cosh (\alpha)-\sinh (\alpha))^{2}} \\
E_{2}= & \frac{2 B r^{*} \cosh (\alpha)}{\operatorname{Da}(\alpha \cosh (\alpha)-\sinh (\alpha))^{2}}-\frac{E_{5}}{D a(\alpha \cosh (\alpha)-\sinh (\alpha))} \\
E_{3}= & \frac{E_{5} \alpha^{2}}{D a(\alpha \cosh (\alpha)-\sinh (\alpha))}-\frac{\alpha^{2} B r^{*} \cosh ^{2}(\alpha)}{2 D a(\alpha \cosh (\alpha)-\sinh (\alpha))^{2}} \\
& +\frac{\alpha^{2} B r^{*}\left(\alpha^{2} D a-1\right)}{(\alpha \cosh (\alpha)-\sinh (\alpha))^{2}} \\
E_{4}= & -E_{1} \cosh (2 \alpha)-E_{2} \cosh (\alpha)-E_{3} \\
E_{5}= & \frac{D a+F(\alpha) B r^{*}+G(\alpha) B r^{*} D a}{\alpha}
\end{aligned}
$$

The expression for the Nusselt number $\mathrm{Nu}$ has been found to be given by

$$
\begin{aligned}
N u= & {[\alpha \cosh (\alpha)-\sinh (\alpha)] /\left[\alpha ^ { 3 } \left(P_{1} \sinh (2 \alpha)+P_{2} \sinh (3 \alpha)\right.\right.} \\
& \left.\left.+P_{3} \sinh (\alpha)+P_{4}\right)\right]
\end{aligned}
$$

where $P_{1}, P_{2}, P_{3}$ and $P_{4}$ are given by

$$
\begin{aligned}
& P_{1}=\frac{E_{1}}{2 \alpha^{3}}-\frac{E_{2}}{4 \alpha^{3} \cosh (\alpha)} \\
& P_{2}=\frac{-E_{1}}{6 \alpha^{3} \cosh (\alpha)} \\
& P_{3}=\frac{E_{2}}{\alpha^{3}}-\frac{E_{1}}{2 \alpha^{3} \cosh (\alpha)}-\frac{E_{3}\left(2+\alpha^{2}\right)}{\alpha^{5} \cosh (\alpha)}-\frac{E_{4}}{\alpha^{3} \cosh (\alpha)} \\
& P_{4}=\frac{2 E_{3}}{\alpha^{4}}+\frac{E_{3}+3 E_{4}}{3 \alpha^{2}}-\frac{E_{2}}{2 \alpha^{2} \cosh (\alpha)}
\end{aligned}
$$

The values of $E_{1}, E_{2}, E_{3}$ and $E_{4}$ are given above.

\section{References}

[1] D.A. Nield, A. Bejan, Convection in Porous Media. Springer, New York, 2006.

[2] D.B. Ingham, I. Pop (Eds.), Transport Phenomena in Porous Media, Elsevier, Oxford, 2005.

[3] K. Vafai (Ed.), Handbook of Porous Media, Taylor \& Francis, New York, 2005.

[4] I. Pop, D.B. Ingham, Convective Heat Transfer: Mathematical and Computational Modelling of Viscous Fluids and Porous Media. Pergamon, Oxford, 2001.

[5] A. Bejan, I. Dincer, S. Lorente, A.F. Miguel, A.H. Reis, Porous and Complex Flow Structures in Modern Technologies. Springer, New York, 2004. 
[6] P. Vadasz (Ed.), Emerging Topics in Heat and Mass Transfer in Porous Media, Springer, New York, 2008

[7] S.D. Harris, D.B. Ingham, I. Pop, Mixed convection boundary-layer flow near the stagnation point on a vertical surface in a porous medium: Brinkman model with slip, Transport Porous Media 77 (2009) 267-285.

[8] A.V. Kuznetsov, D.A. Nield, Thermal instability in a porous medium layer saturated by a nanofluid: Brinkman model, Transport Porous Media. doi:10.1007/s11242-009-9413-2.

[9] L. Storesletten, Effects of anisotropy on convective flow through porous media. in: D.B. Ingham, I. Pop (Eds.), Transport Phenomena in Porous Media. Pergamon, Oxford, 1998, pp. 261-283.

[10] J. Bear, Dynamics of Fluids in Porous Media. Elsevier, New York, 1972.

[11] G. Castinel, M. Combarnous, Critère d'approximation de la convection naturelle dans une couche poreuse anisotrope horizontale, C.R. Acad. Sci. B 278 (1974) 701-704.

[12] J.P. Epherre, Critère d'apparition de la convection naturelle dans une couche poreuse anisotrope, Rev. Gen. Therm. 168 (1975) 949-950.

[13] P.O. Kvernvold, P.A. Tyvand, Nonlinear thermal convection in anisotropic porous media, J. Fluid Mech. 90 (1979) 609-624.

[14] R. McKibbin, Thermal convection in a porous layer: effects of anisotropy and surface boundary conditions, Transport Porous Media 1 (1984) 271-292.

[15] G. Degan, P. Vasseur, E. Bilgen, Convective heat transfer in a vertical anisotropic porous layer, Int. J. Heat Mass Transf. 38 (1993) 1975-1987.

[16] X.L. Zhang, T.H. Nguyen, R. Kahawita, Effects of anisotropy in permeability on the two-phase flow and heat transfer in a porous medium, Heat Mass Transf. 32 (1997) 167-174.

[17] P. Bera, V. Eswaran, P. Singh, Numerical study of heat and mass transfer in an anisotropic porous enclosure due to constant heating and cooling, Numer. Heat Trans. A 34 (1998) 887-905.

[18] P. Bera, A. Khalili, Double-diffusive natural convection in an anisotropic porous cavity with opposing buoyancy forces: multi-solutions and oscillations, Int. J. Heat Mass Transf. 45 (2002) 3205-3222.
[19] A. Kumar, P. Bera, Natural convection in an anisotropic porous enclosure due to nonuniform heating from the bottom wall, ASME J. Heat Transf. 131 (2009) 072601-1-072601-13.

[20] G. Degan, P. Vasseur, Aiding mixed convection through a vertical anisotropic porous channel with oblique principal axes, Int. J. Eng. Sci. 40 (2002) 193-209.

[21] G. Degan, H. Beji, P. Vasseur, L. Robillard, Effect of anisotropy on the development of convective boundary layer flow in porous media, Int. Commun. Heat Mass Transf. 25 (1998) 1159-1168.

[22] P. Vasseur, G. Degan, Free convection along a vertical heated plate in a porous medium with anisotropic permeability, Int. J. Numer. Methods Heat Fluid Flow 8 (1998) 43-63.

[23] G. Degan, C. Akowanou, N.C. Awanou, Transient natural convection of nonNewtonian fluids about a vertical surface embedded in an anisotropic porous medium, Int. J. Heat Mass Transf. 50 (2007) 4629-4639.

[24] D.A.S. Rees, L. Storesletten, The effect of anisotropic permeability on free convective boundary layer flow in porous media, Transport Porous Media 19 (1995) 79-92.

[25] L. Storesletten, D.A.S. Rees, An analytical study of free convective boundary layer flow in porous media: the effect of anisotropic diffusivity, Transport Porous Media 27 (1997) 289-304.

[26] D.A.S. Rees, L. Storesletten, The linear instability of a thermal boundary layer with suction in an anisotropic porous medium, Fluid Dyn. Res. 30 (2002) 155-168.

[27] D.A.S. Rees, L. Storesletten, A.P. Basson, Convective plume paths in anisotropic porous media, Transport Porous Media 49 (2002) 9-25.

[28] Y.-M. Hung, C.P. Tso, Temperature variations of forced convection in porous media for heating and cooling processes: internal heating effect of viscous dissipation, Transport Porous Media 75 (2008) 319-332.

[29] Y.-M. Hung, C.P. Tso, Effects of viscous dissipation on fully developed forced convection in porous media, Int. Commun. Heat Mass Transf. 36 (2009) 597-603.

[30] A.K. Al-Hadhrami, L. Elliott, D.B. Ingham, Combined free and forced convection in vertical channels of porous media, Transport Porous Media 49 (2002) 265-289.

[31] W. Kays, M. Crawford, B. Weigand, Convective Heat and Mass Transfer. McGraw Hill, New York, 2005. 